\title{
The Impact of Transaction Costs on State-Contingent Claims Mispricing
}

\author{
Valerio Restocchi ${ }^{\mathrm{a}, \mathrm{b}}$, Frank McGroarty ${ }^{\mathrm{a}}$, Enrico Gerding ${ }^{\mathrm{b}}$, Johnnie E. V. \\ Johnson $^{\text {a,* }}$ \\ ${ }^{a}$ Southampton Business School, University of Southampton \\ ${ }^{b}$ Electronics and Computer Science, University of Southampton
}

\begin{abstract}
We analyze the impact that transaction costs have on asset mispricing in state-contingent claims markets. In particular, we examine betting markets, in which, it has been argued, transaction costs cause the favorite-longshot bias, a pricing anomaly analogous to the volatility smile in options markets. By using a heterogeneous agents model, we prove that transaction costs alone cannot cause mispricing. Also, we run agent-based simulations to characterize the response of market prices to increments in transaction costs. We find that transaction costs have a significant impact on market inefficiency, by amplifying existing mispricing both directly, influencing market prices, and indirectly, inducing a non-linear response from the agents.
\end{abstract}

Keywords: transaction costs; pricing anomalies; heterogeneous agents; state-contingent claims; favorite-longshot bias.

JEL Classifications: D4, G1

\section{Introduction}

Sports betting markets are a good proxy for financial markets and have been extensively used to analyze market efficiency. In particular, many number of studies have examined the favorite-longshot bias (FLB), an empirical regularity whereby betting on favorites yields higher expected returns than betting on longshots. This anomaly is of noteworthy importance, as it has

\footnotetext{
${ }^{*}$ Corresponding author

Email addresses: vr2g14@soton.ac.uk (Valerio Restocchi), f.j.mcgroarty@soton.ac.uk (Frank McGroarty), eg@ecs.soton.ac.uk (Enrico Gerding), j.e.johnson@soton.ac.uk (Johnnie E. V. Johnson)
} 
been found in most state-contingent claims markets, such as prediction markets (Wolfers and Zitzewitz, 2004) and options markets (Hodges, Tompkins, and Ziemba, 2003). It has also been observed in other contexts (Vaughan Williams et al., 2016).

One of the several theories proposed to explain the FLB argues that transaction costs cause the bias. Hurley and McDonough (1996) suggest that, in a parimutuel market with short-selling constraints, positive transaction costs cause the FLB. However, recent findings demonstrate the need for significant adjustments to their model. In fact, despite the possibility of short-selling in exchange markets, the FLB still exists (Smith, Paton, and Vaughan Williams, 2006). However, both transaction costs and the level of the FLB are significantly lower in such markets, suggesting correlation between the two.

We show how a model in which traders have heterogeneous behaviors can explain all these observations. To achieve this, we extend the model developed by Restocchi et al. (2016) by incorporating transaction costs. We prove that, in a market with a book-balancing bookmaker playing against heterogeneous agents, transaction costs cannot cause the FLB. Then, we investigate price formation by using agent-based simulations. We reproduce three different levels of FLB, including its negative form, and analyze prices under different values of transaction costs. Our results suggest that transaction costs amplify the mispricing generated by the agents' sub-optimal behavior, and that they are positively correlated with the absolute level of the FLB (i.e. with market inefficiency). This finding suggests that, in contrast to the arguments of Hurley and McDonough (1996) and Vaughan Williams and Paton (1998), transaction costs are not correlated with the direction of the FLB.

\section{Model and analysis}

We consider the model introduced by Restocchi et al. (2016), in which agents belonging to five different behavioral classes bet on a two-outcome event in a fixed-odds market. Four of the five types of agents bet according to their utility functions, which are derived from prospect theory, and represent risk loving, risk averse, misperceiving, and informed bettors. The fifth class represents noise bettors, who randomly bet on either outcome A or B. Given the outcome $i \in\{A, B\}$, we denote with $p_{i}$ its probability to happen, with $\pi_{i}$ the associated price of an Arrow-Debreu contract set by the bookmaker, and with $V_{i}$ the fraction of money bet on such outcome (i.e. we assume that the total money bet, $V$, is $V=V_{A}+V_{B}=1$ ). Then, the expected profit for 
the bookmaker is:

$$
\mathbb{E}(P)=V-\frac{p_{A}}{\pi_{A}} V_{A}-\frac{p_{B}}{\pi_{B}} V_{B}
$$

Then, the profit in the single game is defined in the following way:

$$
P= \begin{cases}1-\frac{V_{A}}{\pi_{A}} & \text { if A occurs } \\ 1-\frac{V_{B}}{\pi_{B}} & \text { if B occurs }\end{cases}
$$

Restocchi et al. (2016) take into account two possible pricing strategies that the bookmaker can employ, namely a profit maximization and a risk minimization strategy. Their results, obtained by using both historical data and simulations, suggest that the average bookmaker is more likely to minimize the risk (cf. maximize the profit). This behavior is similar to that of market makers in option pricing, as suggested in the relevant literature. Given the assumption that bookmakers seeks risk minimization, their optimal pricing strategy is to set both potential payoffs to be equal, i.e. $1-\frac{V_{A}}{\pi_{A}}=1-\frac{V_{B}}{\pi_{B}}=\lambda$. By substituting this expression in Eq. (2), we obtain the expression of the prices:

$$
\left\{\begin{array}{l}
\pi_{A}=\frac{V_{A}}{1-\lambda} \\
\pi_{B}=\frac{V_{B}}{1-\lambda}
\end{array}\right.
$$

The profit $\lambda$ is linked to transaction costs by the following relation:

$$
T C=\pi_{A}+\pi_{B}-1=\frac{1}{1-\lambda}-1
$$

Now, we formulate the following proposition:

Proposition 1. Transaction costs are not a sufficient condition to cause the FLB.

In a two-outcome market the FLB exists if and only if the expected return of a bet on $\mathrm{A}$ is higher than that of a bet on $\mathrm{B}$. Hence:

$$
\frac{p_{A}}{p_{B}}>\frac{\pi_{A}}{\pi_{B}} \Leftrightarrow p_{A}>p_{B}
$$

By substituting Eq. (3) in Eq. (5), we obtain the following condition for the FLB to exist:

$$
\frac{p_{A}}{p_{B}}>\frac{V_{A}}{1-\lambda} \frac{1-\lambda}{V_{B}}
$$

The terms including the bookmaker's profit $\lambda$ cancel each other out. Hence, assuming a risk-minimizing bookmaker, the FLB (or its reverse counterpart) cannot be caused by transaction costs. 
Table 1: OLS linear regressions for transaction costs with the level of FLB as dependent variable

\begin{tabular}{|c|c|c|c|c|c|c|}
\hline \multirow[b]{2}{*}{ Variable } & \multicolumn{2}{|l|}{ Weak FLB } & \multicolumn{2}{|l|}{$\begin{array}{l}\text { Strong FLB } \\
\end{array}$} & \multicolumn{2}{|c|}{ Weak negative FLB } \\
\hline & Coefficient (std. err.) & $\mathrm{t}$ & Coefficient (std. err.) & $\mathrm{t}$ & Coefficient (std. err.) & $\mathrm{t}$ \\
\hline Intercept & $0.8488(0.034)$ & $24.88^{*}$ & $6.4252(0.064)$ & $100.23^{*}$ & $0.7327(0.029)$ & $25.67^{*}$ \\
\hline $\mathrm{TC}$ & $18.6904(0.120)$ & $155.76^{*}$ & $7.7950(0.225)$ & $34.576^{*}$ & $18.7383(0.100)$ & $186.66^{*}$ \\
\hline Model Statistics & & & & & & \\
\hline Adj. R-squared & 0.998 & & 0.961 & & 0.999 & \\
\hline F-statistic & 24,260 & & 1,196 & & 34,840 & \\
\hline
\end{tabular}

Hypothesis 1. Transaction costs amplify the level of the FLB and, as a consequence, market inefficiency.

To test this hypothesis, we run agent-based simulations using the market compositions (i.e. distribution of agents) obtained by Restocchi et al. (2016) for three markets with very diverse levels of the FLB, and compute market prices for transaction costs in the range $0 \%-50 \%$ by steps of $1 \%$. The resulting price curves are presented in Figure 1 for the market compositions found for the tennis, the under-over 2.5 goals in football, and the baseball market, which display a weak, strong, and weak negative FLB, respectively.

To analyze in more detail the relation between transaction costs and mispricing, we estimate the parameters of the following equation by using an OLS regression for transaction costs, with the level of the FLB as the dependent variable:

$$
L F L B_{i}=\alpha+\beta T C_{i}+\epsilon_{i}
$$

The level of the FLB is measured as the sum of the differences between each price and the corresponding true probability value, as follows:

$$
L F L B=\int_{0}^{1}|\pi(p, \lambda)-p| d p
$$

Our results, displayed in Table 1, suggest that transaction costs have a direct impact on mispricing. In these regressions, the intercept value indicates the level of FLB caused by agent behavior in a market without transaction costs. It is possible to see that regression statistics, especially the $R^{2}$, are surprisingly good. However, the reason of these results can be easily explained. Transaction costs directly increase prices, and consequently increase market inefficiency, which, in our model, is captured by the FLB metric. Thus, the large goodness-of-fit was expected, and not much informative about the non-trivial impact that transaction costs have on prices. Also, prices are generated by an agent-based model, which by definition is a 


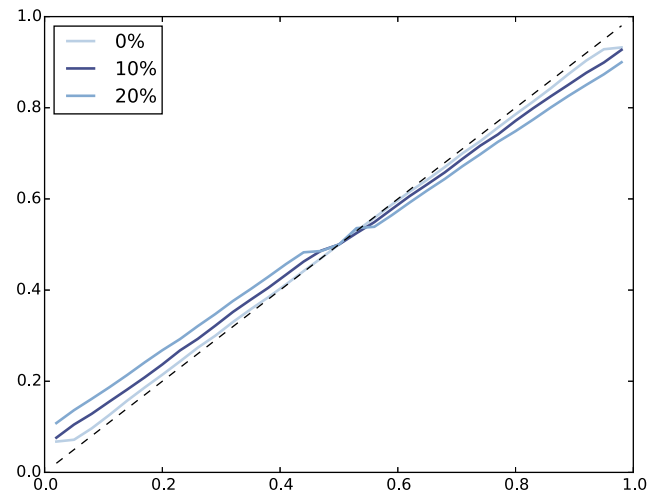

(a)

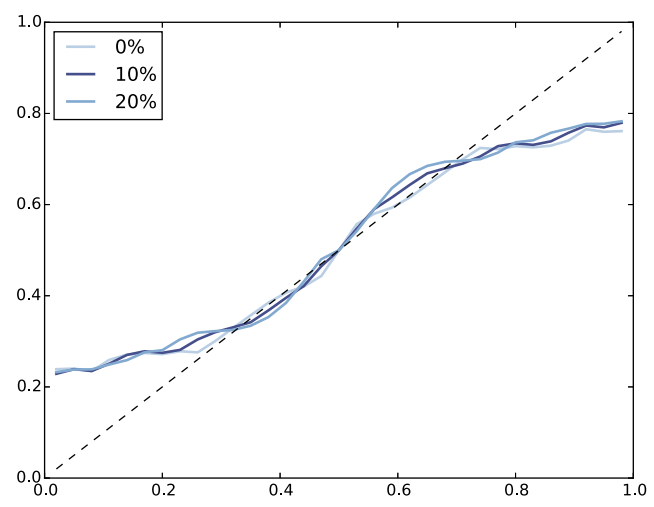

(b)

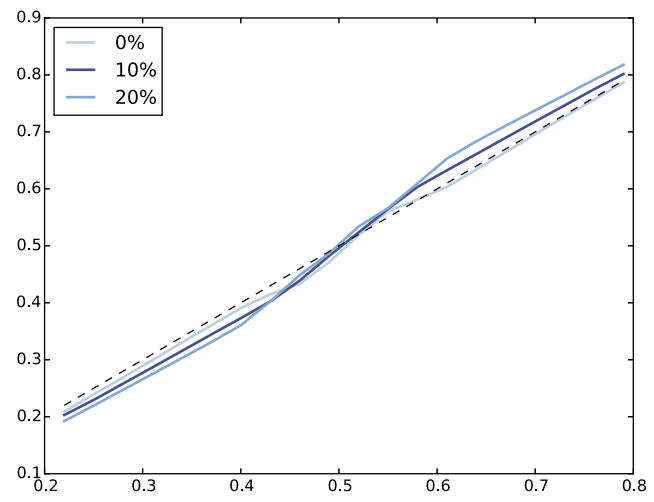

(c)

5

Figure 1: Normalized market price for a given outcome given its probability, generated for the three different markets described by Restocchi et al. (2016). They represent a weak positive (fig.1a), a strong positive (fig.1b), and a weak negative FLB form (fig.1c). Market prices are displayed for transaction costs of $0 \%, 10 \%$, and $20 \%$. 
Table 2: OLS quadratic regressions for transaction costs with the level of FLB after price normalization as dependent variable

\begin{tabular}{lllllll}
\hline & \multicolumn{2}{c}{ Weak FLB } & \multicolumn{2}{c}{ Strong FLB } & \multicolumn{2}{c}{ Weak negative FLB } \\
\hline Variable & Coefficient (std. err.) & $\mathrm{t}$ & Coefficient (std. err.) & $\mathrm{t}$ & Coefficient (std. err.) & $\mathrm{t}$ \\
\hline Intercept & $0.2528(0.015)$ & $17.26^{*}$ & $2.2995(0.014)$ & $162.61^{*}$ & $0.3122(0.018)$ & $37.81^{*}$ \\
TC & $8.1445(0.138)$ & $58.92^{*}$ & $1.1144(0.133)$ & $8.35^{*}$ & $4.1292(0.078)$ & $52.99^{*}$ \\
TC $^{2}$ & $-4.652(0.273)$ & $-16.96^{*}$ & $-2.1902(0.263)$ & $-8.31^{*}$ & $-4.6798(0.154)$ & $-30.43^{*}$ \\
& & & & & & \\
Model Statistics & & & & & & 0.995 \\
\hline Adj. R-squared & 0.998 & & 0.583 & & 4,745 & \\
F-statistic & 14,100 & & & & &
\end{tabular}

* $\quad$ Significant at $0.1 \%$

stylized representation of the market, and might lack of the same sources of noise. However, by examining the regression coefficients for all the markets, we can infer something else.

As shown in Table 1, we find that, for both the positive and negative weak forms of FLB, the coefficients of both $T C$ and the intercepts are similar, and are all largely significant, which suggests that transaction costs are not correlated with the direction of the FLB, but only amplifies its size. Otherwise, the negative FLB would become positive in presence of sufficiently high transaction costs. This result is in contrast to what argued by Hurley and McDonough (1996) and Vaughan Williams and Paton (1998), who suggested that high transaction costs can turn a negative FLB into a positive FLB.

From the results in Table 1 it is also possible to see that, in the market displaying a strong form FLB, the base level of inefficiency is much higher, but the impact of transaction costs on mispricing is lower than in the other two cases. Both these observations are consistent with the fact that, in this market, $45 \%$ of the money is wagered by noise traders (Restocchi et al., 2016). That is, noise traders generate a higher level of mispricing compared with more rational agents, but do not respond to increments in transaction costs, since they continue to trade randomly.

This substantial difference between the coefficients of the variables for the strong form FLB and the two weak ones, which possess a similar market composition, suggests that diverse market compositions respond in considerably different ways to increments of transaction cost values. To investigate this further we develop a quadratic regression to find the relation between transaction costs and normalized market prices. That is, by discounting for transaction costs and then computing the level of FLB, the direct contribution of transaction costs to prices is neglected. Therefore, it is possible to find the mispricing generated by the agents due to a variation of transaction 
costs:

$$
L F L B_{i}=\alpha+\beta T C_{i}+\gamma T C_{i}^{2}+\epsilon_{i}
$$

As shown in Table 2, a quadratic equation fits very well the response of the agents to changes in transaction costs. Once again, we find that the $R^{2}$ are very close to 1, except from that of the strong form FLB regression. This provides additional evidence for our argument. That is, that, in evaluating the goodness-of-fit of these regressions, one should consider that they are performed on simulated data. Indeed, for the strong-form FLB market, which is significantly populated with noise traders, compared with the other markets, the $R^{2}$ is considerably lower. However, once again, the regression coefficients are informative.

In these regressions, the coefficients retain the same sign across all the data sets (positive for the intercept and the first-order term, negative for the second-order term). This implies that, regardless of the market composition, the supplementary inefficiency introduced by the agents always follows a downward concave curve. One possible explanation is that increasing transaction costs progressively deter the most informed bettors. Then, when transaction costs are high and only the least informed bettors are left in the market, the extent of mispricing provides informed bettors with profitable trading opportunities. Consequently, they push prices towards their fundamental values, thus reducing inefficiency.

\section{Conclusion and future work}

We used agent-based modeling to study the effect of transaction costs on asset mispricing. We find that, in a market in which the bookmaker minimizes risk, transaction costs alone cannot generate mispricing. However, our empirical results show that transaction costs significantly affect market prices, amplifying the effect of any existing FLB, but not affecting its direction. Furthermore, we find that transaction costs also augment market inefficiency indirectly by affecting the response of agents, increasing mispricing to an even greater extent.

We believe that the agents' response to changes in transaction costs, and the price inefficiency it generates, is worth further analysis. For instance, such a study could serve to design optimal taxation policies tailored to specific markets, by finding the best tax value for a given market composition. 


\section{References}

Hodges, Stewart, Robert Tompkins, and William Ziemba. 2003. "The Favorite Longshot Bias in SP500 and FTSE100 Index Futures Options." EFA 2003 Annual Conference Paper No. 135.

Hurley, William and Lawrence McDonough. 1996. "The Favourite-Longshot Bias in Parimutuel Betting: A Clarification of the Explanation That Bettors Like to Bet on Longshots." Economics Letters 52:275-278.

Restocchi, Valerio, Frank McGroarty, Enrico Gerding, and Johnnie E.V. Johnson. 2016. "It Takes All Sorts: A Heterogeneous Agent Explanation of State-Contingent Claims Mispricing." Working Paper Available at SSRN: https://ssrn.com/abstract=2858339 .

Smith, Michael A., David Paton, and Leighton Vaughan Williams. 2006. "Market Efficiency in Person-to-Person Betting." Economica 73 (292):673689.

Vaughan Williams, Leighton and David Paton. 1998. "Why are some favourite-longshot biases positive and others negative?" Applied Economics 30:1505-1510.

Vaughan Williams, Leighton, Ming-Chien Sung, Peter Fraser-Mackenzie, John Peirson, and Johnnie Johnson. 2016. "Towards an Understanding of the Origins of the Favourite-Longshot Bias: Evidence from Online Poker Markets, a Real-money Natural Laboratory." Economica .

Wolfers, Justin and Eric Zitzewitz. 2004. "Prediction Markets." Journal of Economic Perspectives 18 (2):107-126. 